\title{
Real-Time Visualization of Ultrasonography Guided Cubital Tunnel Injection: A Cadaveric Study
}

\author{
Jae Min Kim, M.D., Hyun-Mi Oh, M.D., Min-Wook Kim, M.D., Ph.D.
}

Department of Rehabilitation Medicine, Incheon St. Mary's Hospital, College of Medicine, The Catholic University of Korea, Incheon 403-720, Korea

Objective To describe an ultrasonography-guided technique for cubital tunnel injection.

Method The ulnar nerves from 12 elbows of 6 adult cadavers were scanned, and the cross-sectional areas of the ulnar nerves, cubital tunnel inlets and outlets were measured by using ultrasonography. All elbows were dissected after an ultrasonography-guided dye injection at the inlet of the cubital tunnel. The dissectors evaluated the spread of dye and the coloration of the nerve and remeasured the cross-sectional areas of the cubital tunnel inlets and outlets.

Results After a real-time visualization of an ultrasonography-guided injection, the ulnar nerves were seperated from the medial groove for the ulnar nerve. All the ulnar nerves of the cadavers were successfully colored with the dye, from the inlet to oulet of the cubital tunnel. The post-injection cross-sectional areas were significantly larger than the pre-injection cross-sectional areas. No significant differences were detected in the post-injection crosssectional areas of the cubital tunnel outlet and the ulnar nerve as compared with the pre-injection areas.

Conclusion Clinicians should consider real-time visualization of ultrasonography for guided injection around the ulnar nerve at the inlet of the cubital tunnel.

Key Words Cadavers, Ulnar neuropathy, Ultrasonography, Injection

\section{INTRODUCTION}

Ulnar nerve compression at the elbow, manifesting as

Received May 30, 2012; Accepted June 13, 2012

Corresponding author: Hyun-Mi Oh

Department of Rehabilitation Medicine, Incheon St. Mary's Hospital, College of Medicine, The Catholic University of Korea, 56, Dongsu-ro, Bupyeong-gu, Incheon 403-720, Korea

Tel: +82-32-249-7650, Fax: +82-32-280-5040, E-mail: haz521@gmail.com (c) This is an open-access article distributed under the terms of the Creative Commons Attribution Non-Commercial License (http:// creativecommons.org/licenses/by-nc/3.0) which permits unrestricted noncommercial use, distribution, and reproduction in any medium, provided the original work is properly cited.

Copyright $\odot 2012$ by Korean Academy of Rehabilitation Medicine cubital tunnel syndrome, is the second most common entrapment neuropathy in the upper extremity. ${ }^{1}$ It may occur from external compression during repeated elbow flexion. When the elbow is flexed, the tunnel shape changes and pressure on the ulnar nerve is increased, which may lead to ulnar neuropathy. ${ }^{1}$

Clinicians may suspect ulnar neuropathy at the elbow due to characteristic symptoms and signs and can confirm it through electrophysiological studies. Recently, ultrasonography of the ulnar nerve at the elbow has been used to confirm ulnar neuropathy. Ultrasonography is the most commonly used imaging modality because it is inexpensive, real-time, widely available, and flexible. ${ }^{2}$ 
Ulnar neuropathy at the elbow may be treated by either conservative intervention or surgery. As with corticosteroid injection therapy for carpal tunnel syndrome, there is no definite indication for the injection of a corticosteroid in patients with cubital tunnel syndrome. Recently, a few papers ${ }^{3,4}$ were submitted that discuss corticosteriod injection for ulnar neuropathy at the elbow. But previously published descriptions ${ }^{4}$ used an out-of-plane technique near the site of maximal swelling and near the thickest part for injection in the longitudinal plane.

The aim of the present study was to describe a new inplane cubital tunnel injection technique using the transverse plane. It was also conducted to describe a cadaveric study of injection accuracy and to evaluate dynamic anatomic changes and size differences in the cubital tunnel after real-time visualization of an ultrasonography-guided cubital tunnel injection as a non-surgical treatment.

\section{MATERIALS AND METHODS}

We performed ultrasonography on 6 consecutive cadavers (4 men, 2 women; mean age, 71 years; range, 6681 years) with bilateral ulnar nerves at the elbows. Prior to the collection of data, this protocol was approved by our institutional review board.

Ultrasonography was performed with an Accuvix V20 ${ }^{\circledR}$ machine (Medison, Seoul, Korea) with a $12 \mathrm{MHz}$ linear array transducer. Each subject was placed in a prone position and the extended elbow was imaged. The crosssectional area (CSA) of the ulnar nerve, cubital tunnel inlet and outlet were measured. The inlet of the cubital tunnel was defined as the triangular space bordered by the medial epicondyle, the olecranon, and the hyperechoic Osborne's band. The outlet of the tunnel was defined as the space bordered by the flexor carpi ulnaris heads, the ulna and the level where the two heads of the flexor carpi ulnaris joined. ${ }^{2}$

The CSAs of the ulnar nerve and cubital tunnel were measured by tracing along the hyperechoic rim of the nerve and tunnel using the continuous trace function on the ultrasound device. Each measurement was taken twice and the average was recorded. Then all cadavers received an ultrasound-guided injection of $2 \mathrm{ml}$ red dye adjacent to the ulnar nerve at the inlet of the cubital tunnel between the medial epicondyle and ulnar nerve.

The cadaver was prone, with the elbow extended and the forearm supinated. The cubital tunnel was scanned with the transducer to identify the location of the ulnar nerve and cubital tunnel contents as well as any structural abnormalities. The probe was then placed transversely at the inlet of the cubital tunnel while keeping the ulnar nerve. Although the optimal therapeutic site for cubital tunnel injection has not been determined, we prefer to surround the ulnar nerve at the inlet.

Typically, a 25-gauge needle was used for the procedure. The needle passed the medial epicondyle, penetrating the Osborne band between the medial epicondyle and the ulnar nerve at the inlet of cubital tunnel. Under real-time visualization of ultrasonography, the needle was directed to the groove of the ulnar nerve after the dye was delivered. This hydrodissection may separate the medial side of the groove and the ulnar nerve (Fig. 1).

The post-injection CSA of the cubital tunnel inlet, outlet and ulnar nerve were measured using ultrasonography as mentioned earlier. Then, the spread of dye and nerve coloration were evaluated by dissection.

Statistical analyses were performed with SPSS 16.0. All parameters were calculated based on the mean and standard deviation. Comparisons were made using a Wilcoxon signed-rank test, and p-values less than 0.05 were considered significant.
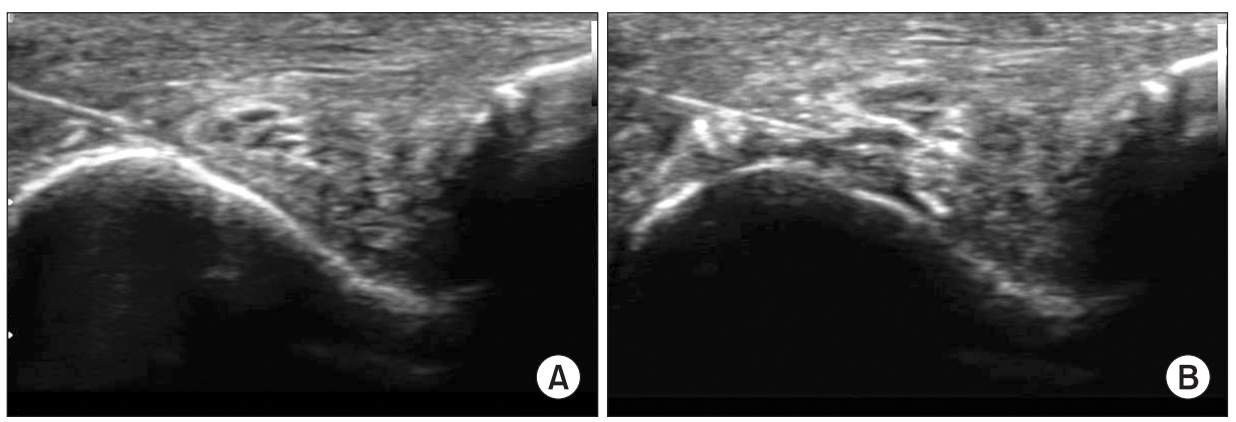

Fig. 1. Transverse scan of the cubital groove. The needle passed the medial epicondyle, penetrating the Osborne bone between the medial epicondyle and ulnar nerve at the inlet of the cubital tunnel, where most ulnar nerve friction occurs. 


\section{RESULTS}

Under real-time visualization of ultrasonography-guided injection, the ulnar nerve was successfully separated from the medial epicondyle and adjacent connective tissues.

In Fig. 1, the shape or CSA of the ulnar nerve was not different after the initial needle injection, and it was well separated from the adjacent connective tissues.

The spread of dye and nerve coloration were evaluated by dissection. At first, the red dye was found between the cubital tunnel inlet and outlet, and as we dissected deeper, dye was spread all over the cubital tunnel from inlet to outlet, and the ulnar nerves were successfully colored with the dye (Fig. 2).

The pre-injection and post-injection cross-sectional areas of the cubital tunnel inlet, outlet and ulnar nerves were measured as previously mentioned. The comparison was made using the Wilcoxon signed-rank test, and p-values of 0.05 were considered significant (Table 1).

The post-injection cross-sectional areas of the cubital tunnel inlet were significantly larger than the pre- injection CSA $(1.07 \pm 0.25,0.91 \pm 0.20, \mathrm{p}<0.05)$. On the ultrasonography, the post-injection CSA of the cubital tunnel outlet seemed increased, but overall, there was no significant difference between the pre-injection and post- injection CSA of the cubital tunnel outlet and the ulnar nerve.

\section{DISCUSSION}

The primary purpose of this report was to describe a new approach for ultrasonography-guided cubital tunnel injection using in-plane technique and to evaluate dynamic anatomic changes.

This technique is similar to the approach for ultrasonography-guided carpal tunnel injection by hydrodissection. In 2008, Smith et al. ${ }^{5}$ used a real-time ultrasoundguided carpal tunnel injection for non-surgical treatment of carpal tunnel syndrome. As they delivered the injection, the nerve was separated from the underlying subsynovial connective tissue, which has recently been implicated in the pathophysiology of idiopathic carpal tunnel syndrome. ${ }^{5}$ This process was named hydrodissection. During the injection, the hydrodissection disrupted adhesions between the median nerve in carpal tunnel and the adjacent connective tissues and allowed the injection material to encircle the target nerve. ${ }^{6}$

In ulnar neuropathy, the authors expected a similar pathophysiology. The reason to inject between the medial epicondyle and ulnar nerve was that the ulnar neuropathy at the elbow may occur from external com-
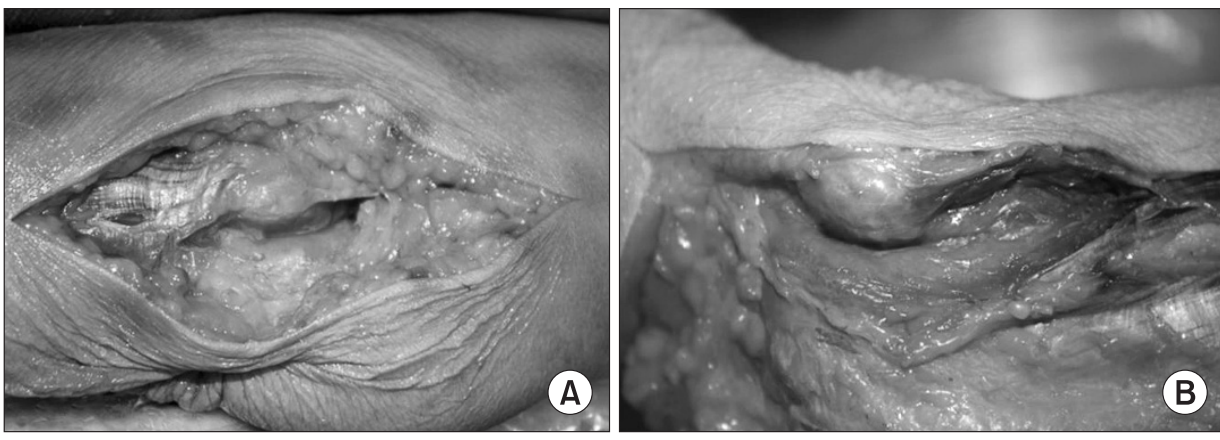

Fig. 2. The Spread of dye and nerve coloration after the injection. It was evaluated by dissection. (A) At first, the red dye was found between the cubital tunnel inlet and outlet, and as we dissected deeper, (B) it spread all over the cubital tunnel from the inlet to the outlet, and the ulnar nerves were successfully colored with dye.

Table 1. The Mean Pre-injection and Post-injection CSAs of the Ulnar Nerve and the Cubital Tunnel at the Inlet and Outlet of the Tunnel

\begin{tabular}{lcccc}
\hline & $\begin{array}{c}\text { Inlet before } \\
\text { injection }\end{array}$ & $\begin{array}{c}\text { Inlet after } \\
\text { injection }\end{array}$ & $\begin{array}{c}\text { Outlet before } \\
\text { injection }\end{array}$ & $\begin{array}{c}\text { Outlet after } \\
\text { injection }\end{array}$ \\
\hline Ulnar nerve CSA $\left(\mathrm{cm}^{2}\right)$ & $0.045 \pm 0.020$ & $0.045 \pm 0.018$ & $0.037 \pm 0.013$ & $0.037 \pm 0.013$ \\
Cubital tunnel CSA $\left(\mathrm{cm}^{2}\right)$ & $0.91 \pm 0.20$ & $1.07 \pm 0.25^{*}$ & $0.14 \pm 0.08$ & $0.17 \pm 0.12$ \\
\hline
\end{tabular}

The values are given as the mean and standard deviation

CSA: Cross-sectional areas

${ }^{*} \mathrm{p}<0.05$ 
pression during repeated elbow flexion, causing ulnar nerve subluxation or dislocation. Repetitive movements of the elbow may result in inflammation, and edema may eventually lead to chronic changes in the nerve. ${ }^{5}$ In 2008 , Yoon et al. ${ }^{2}$ published that the cubital tunnel is a flexible compartment that expands as the ulnar nerve enlarges during entrapment. Therefore, we injected $2 \mathrm{ml}$ of dye between the medial epicondyle and the ulnar nerve, expecting the change flexibility of the cubital tunnel. Under real-time visualization, the injectate was delivered, separating the ulnar nerve from the surrounding connective tissues, and the post-injection CSA of the cubital tunnel outlet seemed increased, while there was no significant difference between the pre-injection and post-injection CSA of the ulnar nerve. This hydrodissection may disrupt adhesion in cubital tunnel syndrome.

There are few reports on injection of a steroid for cubital tunnel syndrome, and the effects of steroid injection are controversial. In 1980, Pechan and Kredba demonstrated that steroid injection is an effective treatment for cubital tunnel syndrome as judged by the symptoms, signs and electrophysiological examinations. ${ }^{7}$ After corticosteroid administration, the amplitude of SNAP is 8.4 $\mu \mathrm{V}$ higher and the conduction velocity is $5.4 \mathrm{~m} / \mathrm{s}$ greater, on average. ${ }^{7}$ Also, on long-term follow-up, symptoms and electrophysiological testing results improved in $67.3 \%{ }^{8}$ However, in 1996, Hong et al. ${ }^{6}$ published that splint application alone was adequate to improve the symptoms of pain and the conduction velocity of the ulnar nerve, demonstrating that splinting assists in keeping the elbow extended or minimally flexed to minimize stretching of the ulnar nerve. It helps to avoid repetitive movement of the elbow which may result in nerve inflammation and edema, eventually causing cubital tunnel syndrome. However, the addition of a steroid injection did not provide a significant change in the treatment of cubital tunnel syndrome. ${ }^{6}$

Very recently a few studies were published, demonstrating the effects of corticosteroid injection for ulnar neuropathy. Alblas et al. ${ }^{3}$ published that injection with a corticosteroid for ulnar neuropathy at the elbow was safe and effective, improving clinical symptoms, and in Rampen's study, ${ }^{4}$ there was also clinical and ultrasonographic improvement in patients with mild ulnar neuropathy at the elbow. But previously published descriptions use an out-of-plane technique near the site of maximal swelling with injection near the thickest part in the longitudinal plane. In this study, the in-plane technique was used of the needle being inserted in the plane of the ultrasonography beam. This technique provides several advantages when compared with previous studies. First, the needle tip and shaft passage can be visualized in-plane relatively to the transducer between the medial epicondyle and the ulnar nerve where ulnar nerve friction mostly occurs. Therefore, it is possible to follow the advancing needle in real time and easier to control the needle. Second, ultrasonography can precisely show periligamentous or periarticular structures as well as anatomic variations; therefore, it can provide accurate injection sites, avoiding the ulnar nerve. Third, vascular structures such as ulnar collateral and recurrent arteries can be avoided.

\section{CONCLUSION}

The present report describes a cadaveric study of injection accuracy and evaluates dynamic anatomic changes and size differences in the cubital tunnel after real-time visualization of ultrasonography-guided cubital tunnel injection as a non-surgical treatment.

We recommend using real-time visualization by ultrasonography for guided injection around the ulnar nerve at the inlet of the cubital tunnel.

\section{REFERENCES}

1. Bayrak AO, Bayrak IK, Turker H, Elmali M, Nural MS. Ultrasonography in patients with ulnar neuropathy at the elbow: comparison of cross-sectional area and swelling ratio with electrophysiological severity. Muscle Nerve 2010; 41: 661-666

2. Yoon JS, Hong SJ, Kim BJ, Kim SJ, Kim JM, Walker FO, Cartwright MS. Ulnar nerve and cubital tunnel ultrasound in ulnar neuropathy at the elbow. Arch Phys Med Rehabil 2008; 89: 887-889

3. Alblas CL, van Kasteel V, Jellema K. Injection with corticosteroids (ultrasound guided) in patients with an ulnar neuropathy at the elbow, feasibility study. Eur J Neurol 2012; 16: 1-4

4. Rampen AJ, Wirtz PW, Tavy DL. Ultrasound-guided steroid injection to treat mild ulnar neuropathy at the elbow. Muscle Nerve 2011; 44: 128-130 
5. Smith J, Wisniewski SJ, Finnoff JT, Payne JM. Sonographically guided carpal tunnel injections: the ulnar approach. J Ultrasound Med 2008; 27: 1485-1490

6. Hong CZ, Long HA, Kanakamedala RV, Chang YM, Yates L. Splinting and local steroid injection for the treatment of ulnar neuropathy at the elbow: clinical and electrophysiological evaluation. Arch Phys Med Rehabil 1996; 77: 573-577
7. Pechan J, Kredba J. Treatment of cubital tunnel syndrome by means of local administration of cortisonoids. II. Long-term follow-up. Acta Univ Carol Med (Praha) 1980; 26: 135-140

8. Pechan J, Kredba J. Treatment of cubital tunnel syndrome by means of local administration of corticosteroids. I. Short-term follow-up. Acta Univ Carol Med (Praha) 1980; 26: 125-133 